\title{
O Feto e a Hermenêutica da Diástole
}

\author{
Paulo Zielinsky
}

Porto Alegre, RS

A diástole ventricularé uma fase do ciclo cardíaco em que os eventos se sucedem de forma menos explícita do que na sístole. Nesta, a primeira e fundamental função do coração é a de bombear o sangue para a circulação. Desde o início da ciência, os estudiosos concluíram que a contração desta bomba era uma atividade vital para a manutenção da perfusão dos tecidos, mas foi só recentemente ${ }^{1-5}$ que o enchimento dos ventrículos e sua distensibilidade passaram a ser considerados, também, como funções importantes para a homeostase e a vida.

O reconhecimento das diversas fases da diástole tornou possível a compreensão dos processos fisiológicos e patológicos relacionados com o enchimento ventricular. Assim, surgiram os conceitos de relaxamento e complacência ventricular, rigidez miocárdica e recuo elástico. Cada uma dessas fases tem sido estudada, separadamente e em conjunto, através de inúmeros índices quantitativos, que buscam avaliar a normalidade ou a anormalidade da diástole ventricular. Um número impressionante de publicações inundou, nos últimos anos, a literatura médica internacional, tratando dos fenômenos relacionados com a diástole dos ventrículos, e com o que passou a ser chamado de "função diastólica" e até de "diastologia" ${ }^{6,7}$. A cavidade ventricular mais contemplada com o avanço do conhecimento relativo à diástole foi, sem surpresa, o ventrículo esquerdo, muito especialmente nos trabalhos realizados com adultos. Na área da cardiologia pediátrica, também foram publicados, em muito menor escala, artigos estudando a função diastólica do ventrículo esquerdo, especialmente em relação às alterações conseqüentes a hipertensão arterial e miocardiopatias ${ }^{8,9}$, e do ventrículo direito ${ }^{10}$, no pós-ope-

Instituto de Cardiologia do Rio Grande do Sul/Fundação Universitária de Cardiologia Correspondência: Paulo Zielinsky - Instituto de Cardiologia Fetal do IC/FUC Av. Princesa Isabel, 395 - 90620-001 - Porto Alegre, RS - E-mail: pesquisa@ cardnet.tche.br

Recebido para publicação em 25/7/01

Aceito em 30/4/02 ratório de algumas cardiopatias congênitas e de transplante cardíaco $^{11,12}$. Entre os métodos diagnósticos mais utilizados para quantificar os eventos diastólicos normais e anormais predomina, indiscutivelmente, a Doppler-ecocardiografia, especialmente a análise da curva do fluxo transmitral, para o ventrículo esquerdo, e da do fluxo transtricuspídeo, para o ventrículo direito. O ecocardiograma unidimensional da valva mitral e da parede atrial esquerda tem também sido descrito como capaz de fornecer informações importantes sobre a função diastólica do ventrículo esquerdo ${ }^{11}$.

O estudo da função diastólica do feto tem sido contemplado com relativamente poucos trabalhos, e a grande maioria deles utiliza a análise Doppler-fluxométrica das curvas de velocidade mitral e tricúspide. Já está bem estabelecido que a velocidade do fluxo atrioventricular, tanto direito como esquerdo, é menor na fase de enchimento ventricular passivo (onda "E") do que na fase de enchimento ativo, ou contração atrial (onda "A"), de forma que a relação E/A é $<1$, embora com valores progressivamente crescentes, durante todo o $2^{\circ}$ e o $3^{\circ}$ trimestre da gestação ${ }^{12}$. Alterações na complacência ou no relaxamento ventricular podem alterar esse padrão, e esse dado tem sido usado para quantificar alterações da função diastólica de ambos os ventrículos durante a vida pré-natal ${ }^{13-28}$. Outros índices também derivados das curvas de velocidade atrioventricular têm sido utilizados, como a integral velocidade-tempo, refletindo o comportamento da mesma variável ${ }^{29}$.

A circulação fetal tem características únicas, que tornam insuficientes os métodos tradicionais de avaliação da diástole ventricular utilizados na vida pós-natal, em qualquer idade.

A função diastólica dos dois ventrículos é absolutamente interdependente, sendo o grande elo em comum o forame oval ${ }^{30}$. São elementos fundamentais para a função diastólica do ventrículo esquerdo: o retorno venoso pela veia cava inferior, já que $1 / 3$ do fluxo neste vaso, correspondentes à coluna de sangue mais oxigenado que vem do ducto venoso, atinge as cavidades esquerdas através do forame oval; o tamanho do forame oval; o fluxo venoso 
pulmonar, que é muito menor do que na vida pós-natal, devido à grande resistência vascular pulmonar, mas que pode chegar a $20 \%$ do débito cardíaco; e a função diastólica do ventrículo direito, já que qualquer interferência no enchimento ventricular direito irá refletir-se numa maior pré-carga para o ventrículo esquerdo. Por outro lado, os componentes importantes da função diastólica do ventrículo direito são: 0 fluxo da porção cefálica do feto, através da veia cava superior; o fluxo da veia cava inferior que não foi direcionado para o forame oval; o tamanho do forame oval; e, de forma muito significante, a função diastólica ventricular esquerda, pois qualquer situação que interfira no enchimento do ventrículo esquerdo ou que aumente a pressão atrial esquerda, diminuirá o fluxo interartrial pelo forame oval ${ }^{30-32}$.

O estudo Doppler-fluxométrico da função diastólica do ventrículo direito, desta maneira, pode ser realizado de forma muito mais fácil e acurada do que utilizando a análise do fluxo transtricuspídeo, através da avaliação das curvas de fluxo pelas veias umbilical ${ }^{31}$, cava inferior ${ }^{32-37}$, hepáticas e, especialmente, pelo ducto venoso ${ }^{38}$. A literatura obstétrica tem sido rica em publicações sobre o comportamento Doppler-fluxométrico do sistema venoso fetal, mas esta metodologia tem sido muito pouco descrita na avaliação de situações patológicas da circulação fetal de causa cardiológica. Parece lógico sugerir que a avaliação dos fluxos venosos seja de amplo domínio do cardiologista pediátrico, de forma a incluir esta abordagem na rotina do exame ecocardiográfico fetal. Um grande número de situações estritamente cardiológicas poderá ser melhor equacionado, tanto do ponto de vista propedêutico como de manejo pré e pós-natal imediato, se a função diastólica do ventrículo direito estiver adequadamente estabelecida pela análise dos fluxos venosos. Assim, o aparecimento de pulsações venosas umbilicais, o aumento do fluxo reverso pré-sistólico (onda "A") na veia cava inferior ou nas veias hepáticas, ultrapassando 15\% do componente sistólico (índice de pré-carga) ou a diminuição ou reversão do fluxo pré-sistólico no ducto venoso constituem-se em evidências de disfunção diastólica do ventrículo direito e conseqüente comprometimento hemodinâmico fetal significante ${ }^{33-38}$. Temos observado alterações da função diastólica do ventrículo direito, pela análise do comportamento das curvas do fluxo venoso, em diversas situações clínicas, como compressão extracardíaca, bloqueio atrioventricular total, taquiarritmia supraventricular, cardiopatias estruturais com alterações do enchimento ventricular e muitas outras.

No que se refere à avaliação da função diastólica do ventrículo esquerdo, também se podem aplicar os princípios da hermenêutica, ao se buscar uma releitura de conhecimentos simples para o entendimento de um fenômeno. Ao reconhecer que o forame oval é a mais importante porta de comunicação entre a circulação esquerda e a direita, e de que a mobilidade da membrana que o guarda (o "septum pri- mum") pode refletir modificações relacionadas com a pressão atrial esquerda, sugerimos que alterações na complacência e/ou no relaxamento do ventrículo esquerdo, ao se refletirem na pressão atrial esquerda, devam interferir nesta mobilidade. Assim, levantamos a hipótese de que situações que facilitem o enchimento ventricular esquerdo ( $m a i-$ or complacência e/ou relaxamento) devem aumentar o deslocamento linear do "septum primum" na diástole, por menor pressão atrial esquerda e de que, analogamente, situações que dificultem o enchimento ventricular esquerdo (menor complacência e/ou relaxamento) devem diminuir este deslocamento, por maior pressão atrial esquerda. Para testar estas hipóteses, nosso grupo delineou dois modelos com características funcionais distintas: a respiração fetal, cujo reflexo na melhora da função diastólica ventricular esquerda, por aumento do enchimento ventricular, já havia sido demonstrada, analisando curvas de fluxo transmitral, foi utilizada como modelo fisiológico de aumento da complacência e do relaxamento ventriculares, enquanto que a hipertrofia miocárdica septal em fetos de mães diabéticas foi escolhida como modelo de diminuição da complacência e do relaxamento ventriculares esquerdos. Esta doença tem sido objeto de linha de pesquisa coordenada pelo autor ${ }^{39-44}$. Para a quantificação do deslocamento linear do "septum primum", foi utilizado um índice de excursão", obtido pela razão entre a deslocamento linear máximo do "septum primum" ao final da diástole e o máximo diâmetro atrial esquerdo, ao ecocardiograma fetal bidimensional, em um corte de quatro câmaras ${ }^{45}$. No primeiro trabalho, apresentado no XLII Congresso da Sociedade Brasileira de Cardiologia, em Goiânia ${ }^{46}, 28$ fetos normais foram examinados em apnéia e durante movimentos respiratórios, medindo-se o índice de redundância nos dois momentos. O índice de excursão médio em apnéia foi de $0,39 \pm 0,05(0,29-0,48)$ e durante os movimentos respiratórios foi de $0,57 \pm 0,07(0,45-0,69)$, sendo esta diferença significante $(\mathrm{p}<0,001)$. A conclusão desse estudo foi de que a excursão do "septum primum" é maior durante a respiração do que em apnéia, confirmando a hipótese conceitual. O segundo projeto ${ }^{47^{*}}$ avaliou o índice de excursão em três grupos de fetos: 29 fetos de mães diabéticas com hipertrofia septal, 33 fetos de mães diabéticas sem hipertrofia miocárdica e 35 fetos normais, de mães sem alterações glicêmicas. Após 32 semanas de gestação, observou-se que os fetos de mães diabéticas com hipertrofia miocárdica tinham índice de excursão significativamente menor $(0,33 \pm 0,21)$ do que os do grupo de mães diabéticas sem hipertrofia miocárdica $(0,44 \pm 0,22 ; \mathrm{p}=0,002)$ e os fetos normais $(0,45 \pm 0,19 ; \mathrm{p}=0,003)$; além disso, foi demonstrada correlação inversa significante $(r=-0,7 ; p=0,04)$ entre a espessura septal e o índice de excursão do "septum primum". Não ocorreram modificações nos fluxos através das valvas atrioventriculares. Esses achados também confirmaram a hipótese de que a 
excursão do "septum primum" esteve diminuída num modelo em que existe alteração do enchimento ventricular esquerdo. Diante desses dados, pode-se sugerir que a avaliação da excursão do "septum primum" pode ser utilizada como um índice adicional de avaliação da função diastólica do ventrículo esquerdo. Obviamente, esses achados abrem a perspectiva de uma linha de investigação a ser seguida, e permitem inferências que por sua vez podem ser hipóteses de trabalho para novos projetos. Trabalhos em andamento no nosso grupo relacionados com este tema incluem a avaliação da excursão do "septum primum" no retardo do crescimento intra-uterino, sua utilidade como índice de avaliação funcional durante a transfusão intra-uterina na isoimunização Rh, a relação entre a excursão do "septum primum" e o fluxo no ducto venoso em diversas situações normais e patológicas, a medida da relação entre a área de excursão do "septum primum" e a área atrial esquerda, o comportamento do fluxo no ducto venoso e da excursão do "septum primum" nas cardiopatias estruturais, estudos de coorte de fetos com alterações na excursão do "septum primum" em relação a desfechos pré e pós-natais, a avaliação da função diastólica fetal através da análise do fluxo em veia pulmonar, do encurtamento atrial esquerdo, do fluxo através do forame oval e da movimentação das valvas atrioventriculares e das paredes pelo Doppler tecidual.

Assim, pode-se afirmar que o estudo da função diastólica do feto humano tem passado, presente e futuro, como de resto qualquer área da ciência. É importante manter permeável nosso filtro de informações, assim como atuantes nossos centros integradores e nosso pensamento lateral, de forma a buscar ativamente o avanço do conhecimento e a incorporação de novos valores e paradigmas.

\section{Referências}

1. Dodek A, Kassebaum DG, Bristow JD. Pulmonary edema in coronary-artery disease without cardiomegaly: paradox of the Stiff heart. N Engl J Med 1972; 286: $1347-50$.

2. Danford DA, Huhta JC, Murphy DJ. Doppler echocardiographic approaches to ventricular diastolic function. Echocardiography 1986; 3: 33-40.

3. Hanrath P, Mathey DG, Siegert R, Bleifeld W. Left ventricular relaxation and filling pattern in different forms of left ventricular hypertrophy: an echocardiographic study. Am J Cardiol 1980; 45: 15-23.

4. Gidding SS, Snider AR, Rocchini AP, Peters J, Farnsworth R. Left ventricular diastolic filling in children with hypertrophic cardiomyopathy: assessment with pulsed Doppler echocardiography. J Am Coll Cardiol 1986; 8: 310-6.

5. Maron BJ, Spirito P, Kimberly JG, Wesley YE, Bonow RO, Arce J. Noninvasive assessment of left ventricular diastolic function by pulsed Doppler echocardiography in patients with hypertrophic cardiomyopathy. J Am Coll Cardiol 1987; 10: $733-42$

6. Danford DA, Huhta JC, Murphy DJ. Doppler echocardiographic approaches to ventricular diastolic function. Echocardiography 1986; 3: 33-40.

7. Nishimura RA, Appleton CP. "Diastology": beyond E and A. J Am Coll Cardiol 1996; 27: 372-4.

8. Galderisi M, Celentano A, Tammaro P, et al. Left ventricular hypertrophy, compliance and ventricular filling. J Int Med Res 1991; 19: 103-11.

9. Gidding SS, Snider AR, Rocchini AP, Peters J, Farnsworth R. Left ventricular diastolic filling in children with hypertrophic cardiomyopathy: assessment with pulsed Doppler echocardiography. J Am Coll Cardiol 1986; 8: 310-6.

10. Yu CM, Sanderson JE, Chan S, Yeung L, Hung YT, Woo KS. Right ventricular diastolic dysfunction in heart failure. Circulation 1996; 93: 1509-14.

11. Pahl E, Miller AS, Griffith BP, Fricker FJ. Occult restrictive hemodynamics after pediatric heart transplantation. J Heart Lung Transplant 1995; 14: 1109-15.

12. Rizzo G, Nicolaides KH, Arduini D, Campbell S. Effects of intravascular fetal blood transfusion on fetal intracardiac Doppler velocity waveforms. Am J Obstet Gynecol 1990; 163: 1231-8.

13. Frommelt MA, Snider AR, Crowley DC, Meliones JN, Heidelberger KP. Echocardiographic indexes of allograft rejection in pediatric cardiac transplant recipients. J Am Soc Echocardiogr 1992; 5: 41-7.

14. BriguoriC, Betocchi S, Losi MA, etal. Noninvasive evaluation of left ventriculardiastolic function in hypertrophic cardiomyopathy. Am J Cardiol 1998; 81: 180-7.

15. Tulzer G, Khowsathit $P$, Gudmundsson $S$, et al. Diastolic function of the fetal heart during the second and third trimester: a prospective longitudinal Dopplerechocardiographic study. Eur J Pediatr 1994; 153: 151-4.

16. van der Mooren K, Barendregt LG, Wladimiroff JW. Fetal atrioventricular and outflow tract flow velocity waveforms during normal second half of pregnancy. Am J Obstet Gynecol 1991; 165: 668-74.

17. Weber HS. Serial echocardiographic Doppler evaluation of diastolic function in the normal human fetus. Cardiol Young 1996; 6: 32-6.
18. Weiner Z, EfratZ,ZimmerEZ, Itskovitz-Eldor J. Fetal atrioventricular blood flow throughout gestation. Am J Cardiol 1997; 80: 659-62.

19. Carceller-Blanchard AM, Fouron JC. Determinants of the Doppler flow velocity profile through the mitral valve of the human fetus. Br Heart J 1993; 70: 457-60.

20. Reed KL, Sahn DJ, Scagnelli S, Anderson CF, Shenker L. Doppler echocardiographic studies of diastolic function in the human fetal heart: changes during gestation. J Am Coll Cardiol 1986; 8: 391-5.

21. Hata T, Aoki S, Hata K, Kitao M. Intracardiac blood flow velocity waveforms in normal fetuses in utero. Am J Cardiol 1987; 59: 464-8.

22. Choi JY, Noh CI, Yun YS. Study on Doppler waveforms from the fetal cardiovascular system. Fetal Diagn Ther 1991; 6: 74-83.

23. Harada K, Rice MJ, Shiota T, et al. Gestational age- and growth-related alterations in fetal right and left ventricular diastolic filling patterns. Am J Cardiol 1997; 79: 173-7.

24. Veille JC, Smith N,Zaccaro D. Ventricular filling patterns of the right and left ventricles in normally grown fetuses: a longitudinal follow-study from early intrauterine life to age 1 year. Am J Obstet Gynecol 1999; 180: 849-58.

25. Tsyvian P, Malkin K, Wladimiroff JW. Assessment of fetal left cardiac isovolumic relaxation time in appropriate and small-for-gestational-age fetuses. Ultrasound Med Biol 1995; 21: 739-43.

26. Harada K, Rice MJ, McDonald RW, et al. Doppler echocardiographic evaluation of ventricular diastolic filling in fetuses with ductal constriction. Am J Cardiol 1997; 79: 442-6.

27. Rizzo G, Arduini D, Romanini C, Mancuso S. Doppler echocardiographic assessment of atrioventricular velocity waveforms in normal and small-for-gestational-age fetuses. Br J Obstet Gynaecol 1988; 95: 65-9.

28. Tsyvian P, Malkin K, Artemieva O, WladimiroffJW. Assessment of left ventricular filling in normally grown fetuses, growth-resticted fetuses and fetuses of diabetic mothers. Ultrasound Obstet Gynecol 1998; 12: 33-8.

29. Rizzo G, Arduini D, Romanini C. Doppler echocardiographic assessment of fetal cardiac function. Ultrasound Obstet Gynecol 1992; 2: 434-5.

30. Tulzer G, Khowsathit P, Gudmundsson S, et al. Diastolic function of the fetal heart during the second and third trimester: a prospective longitudinal Dopplerechocardiographic study. Eur J Pediatr 1994; 153: 151-4.

31. Tsyvian P, Malkin K, Artemieva O, WladimiroffJW. Assessment of left ventricular filling in normally grown fetuses, growth-resticted fetuses and fetuses of diabetic mothers. Ultrasound Obstet Gynecol 1998; 12: 33-8.

32. Fouron JC. Fetal cardiovascular physiology. In: Allan LD, Hornberger L, Sharland G. Textbook of Fetal Cardiology. London: Greenwich Medical Media Ltd, 2000; 3: 29-45.

33. Rizzo G, Arduini D, Romanini C. Pulsations in umbilical vein: a physiological finding in early pregnancy. Am J Obstet Gynecol 1992: 167: 675-7.

34. Reed KL, Appleton CP, Anderson CF, Shenker L, Sahn DJ. Doppler studies of 
vena cava flows in human fetuses - insights into normal and abnormal cardiac physiology. Circulation 1990; 81: 498-505.

35. Rizzo G, Capponi A, Talone PE, Arduini D, Romanini C. Doppler indices from inferior vena cava and ductus venosus in predicting $\mathrm{pH}$ and oxygen tension in umbilical blood at cordocentesis in growth retarted fetuses. Ultrasound Obstet Gynecol 1996; 7: 401-10.

36. Kanzaki T, Chiba Y. Evaluation of preload condition of the fetus by inferior vena cava blood flow pattern. Fetal Diagn Ther 1990; 5: 168-74.

37. Okamura K, Murotsuki J, Kobajashi M, Tanigawara S, Uehara S, Yajima A. Umbilical venous pressure and Doppler flow patterns of inferior vena cava in the fetus. Am Perinatal 1994; 11: 255-9.

38. Rizzo G, Capponi A, Arduini D, Romanini C. Ductus venosus velocity waveforms in appropriate and small for gestational age fetuses. Early Hum Dev 1994; 39: 15-26.

39. Zielinsky P. Role of prenatal echocardiography in the study of hypertrophic cardiomyopathy in the fetus. Echocardiography 1991; 8: 661-7.

40. Hagemann LL, Zielinsky P. Estudo pré-natal da miocardiopatia hipertrófica e sua associação com os níveis de insulina em fetos de mães diabéticas. Arq Bras Cardol 1996; 66: 193-8

41. Zielinsky P, Hagemann LL, Daudt LE, Behle I. Pre and postnatal analysis of fac- tors associated with fetal myocardial hypertrophy in diabetic pregnancies. J Matern Fetal Invest 1992; 2: 163-7.

42. Behle I, Zielinsky P, Zimmer L, Pontremoli M, Rsich J. Níveis de hemoglobina glicosilada e anomalias cardíacas em fetos de mães com diabetes mellitus. Rev Bras Ginecol Obstet 1998; 5: 237-43.

43. Zielinsky P, Costa MHL, Oliveira LT, Bonow FP, Silva NIT, Hageman LL. Estudo da história natural da hipertrofia miocárdica e sua associação com hiperinsulinismo em filhos de mães diabéticas. Arq Bras Cardiol 1997; 69: 389-94.

44. Menezes HS, Barra M, Belló A, Martins CB, Zielinsky P. Fetal myocardial hypertrophy in a experimental model of gestational diabetes. Cardiol Young 2001; 11: 609-13

45. Firpo C, Zielinsky P. Mobility of the flap valve of the primary atrial septum in the developing human fetus. Cardiol Young 1998; 8: 67-70.

46. Zielinsky P, Mastalir E, Antunes P, et al. Comportamento do septum primum durante a respiração fetal: um modelo fisiológico para avaliar a função diastólica ventricular esquerda? Arq Bras Cardiol 2001; 77(supl. I): 59.

47. Firpo C, Zielinsky P. Medidas ecocardiográficas, fluxos atrioventriculares e mobilidade do "septum primum": modificações evolutivas e implicações funcionais em fetos normais e de mães diabéticas [Tese]. Porto Alegre: Programa de Pós-Graduação/UFRGS, 2000: 160p.

\section{Bula Resumida}

Micardis ${ }^{\circledR}$ HCT - Telmisartam/Hidroclorotiazida - Uso adulto - Composição: Cada comprimido de MICARDIS HCT contém 40 mg/12,5 mg ou 80 mg/12,5 mg de telmisartam/hidroclorotiazida. Excipientes q.s.p. 1 comprimido. Indicações: Tratamento da hipertensão arterial. MICARDIS HCT, como associação de dose fixa, é indicado em pacientes cuja pressão arterial não é adequadamente controlada com telmisartam ou hidroclorotiazida isoladamente. Posologia: MICARDIS HCT deve ser administrado uma vez ao dia. A dose de MICARDIS pode ser aumentada gradativamente antes de substituí-lo pelo MICARDIS HCT. A substituição direta da monoterapia pelas combinações fixas pode ser considerada. MICARDIS HCT $40 / 12,5$ mg pode ser administrado em pacientes cujas pressões sangüíneas não sejam adequadamente controladas por MICARDIS $40 \mathrm{mg}$ ou hidroclorotiazida. MICARDIS HCT $80 / 12,5$ mg pode ser administrado em pacientes cujas pressões arteriais não sejam adequadamente controladas por MICARDIS $80 \mathrm{mg}$ ou por MICARDIS HCT $40 / 12,5 \mathrm{mg}$. $\mathrm{O}$ máximo efeito anti-hipertensivo é obtido após 4 a 8 semanas de tratamento. Quando necessário, MICARDIS HCT pode ser administrado com outros antihipertensivos. Devido ao componente hidroclorotiazida, MICARDIS HCT não deve ser usado em pacientes com disfunção renal grave. Nos casos leves a moderados, não se observaram efeitos adversos renais e não é necessário ajuste de dose. Recomenda-se monitoração periódica da função renal. Nos casos de insuficiência hepática leve a moderada, não se deve exceder a dose de 40/12,5 mg uma vez ao dia. MICARDIS HCT não é indicado em pacientes com insuficiência hepática grave. Não são necessários ajustes de dose conforme a idade. Ainda não se estabeleceram a segurança e a eficácia de MICARDIS HCT em pacientes menores de 18 anos. Contra-indicações: Hipersensibilidade aos componentes ativos, excipientes ou substâncias derivadas de sulfonamidas (como HCT). Gravidez e lactação. Colestase e distúrbios obstrutivos biliares. Insuficiência hepática ou renal grave (depuração de creatinina $<30 \mathrm{ml} / \mathrm{min}$ ). Hipopotassemia refratária e hipercalcemia. Precauções: Não utilizar nos casos de distúrbios biliares obstrutivos ou insuficiência hepática grave. Usar com cautela nos casos de função hepática alterada ou doença hepática progressiva, pois pode ocorrer coma hepático. Há maior risco em pacientes com estenose arterial renal bilateral ou estenose com um único rim funcionando. Não utilizar em insuficiência renal grave, muito cuidado em pacientes com insuficiência renal leve a moderada. Não há experiência em pacientes com rim transplantado. Pode ocorrer hipotensão sintomática em pacientes que têm volemia e/ou sódio depletado. Insuficiência cardíaca congestiva grave ou doença renal, estenose da artéria renal tratada concomitantemente com drogas que afetem o sistema renina-angiotensina-aldosterona foram associados com hipotensão aguda, hiperazotemia, oligúria ou, raramente, insuficiência renal aguda. Aldosteronismo primário: não se recomenda o uso de telmisartam, precaução especial em estenose valvar aórtica ou mitral e cardiomiopatia hipertrófica obstrutiva. Como tiazidas podem prejudicar a tolerância à glicose, pode ser necessário ajuste no tratamento em diabéticos e pode ocorrer manifestação de diabetes latente. A hidroclorotiazida aumenta os níveis de colesterol e triglicérides; contudo, nenhum ou poucos efeitos adversos foram relatados na dose de $12,5 \mathrm{mg}$ contida em MICARDIS HCT. Pode ocorrer hiperuricemia ou precipitação de gota devido a hidroclorotiazida. Pode ser necessária monitoração periódica dos níveis séricos de eletrólitos, uma vez que a hidroclorotiazida pode causar desequilíbrio eletrolítico ou de fluidos (hipopotassemia, hiponatremia e alcalose hipoclorêmica). O tratamento concomitante com telmisartam pode reduzir a hipopotassemia induzida por diuréticos, devido aos efeitos antagônicos. Diuréticos poupadores de potássio, suplementos de potássio devem ser utilizados concomitantemente com cautela. Não há evidências de que MICARDIS HCT reduza ou previna hiponatremia induzida por diuréticos. A deficiência de cloreto é geralmente leve. Tiazidas podem causar elevação dos níveis séricos de cálcio e magnésio. Interromper o uso antes dos testes de função da paratireóide. Não utilizar em intolerância hereditária à frutose. A redução excessiva da pressão arterial no caso de doença cardiovascular isquêmica pode ser prejudicial. Relatou-se exacerbação ou ativação do lupus eritematoso sistêmico com o uso de hidroclorotiazida. Recomenda-se cautela no uso de anti-hipertensivos ao dirigir e operar máquinas. Interações medicamentosas: Muito raramente, relataram-se aumentos das concentrações séricas de lítio. O efeito de depleção de potássio da hidroclorotiazida é atenuado pelo efeito poupador de potássio do telmisartam, mas é potencializado por outros diuréticos caliuréticos, laxantes, anfotericina, carbenoxolona, penicilina G sódica, ácido salicílico e derivados. Por outro lado, o uso concomitante de diuréticos poupadores de potássio, suplementos/sais de potássio ou outras drogas podem aumentar os níveis séricos de potássio. Recomenda-se monitoração periódica de potássio na administração de MICARDIS HCT com digitálicos e antiarrítmicos. Telmisartam pode aumentar o efeito hipotensor de outros agentes anti-hipertensivos. Não se identificaram outras interações de importância clínica. Interações com a hidroclorotiazida: álcool, barbitúricos ou narcóticos (potencialização da hipotensão ortostática); drogas antidiabéticas (ajustes de dose podem ser necessários); colestiramina e resina colestipol (absorção de hidroclorotiazida é prejudicada); corticosteróides, ACTH (depleção eletrolítica, principalmente aumento de hipopotassemia); glicosídeos digitálicos (hipopotassemia ou hipomagnesemia induzida por tiazídicos favorece o aparecimento de arritmias cardiacas induzidas por digitálicos); drogas antiinflamatórias não-esteroidais (a administração de droga antiinflamatória nãoesteroidal pode reduzir os efeitos diurético, natriurético e anti-hipertensivo dos diuréticos tiazídicos em alguns pacientes). Os efeitos das aminas hipertensoras podem ser levemente diminuídos. Os relaxantes musculares esqueléticos não-despolarizantes são potencializados pela hidroclorotiazida. Medicamentos uricosúricos podem requerer ajustes. Verificou-se aumento da incidência de reações de hipersensibilidade ao alopurinol. Os efeitos hiperglicêmicos dos betabloqueadores e diazóxido podem ser aumentados pelas tiazidas. Os agentes anticolinérgicos (por exemplo, atropina, biperideno) podem aumentar a biodisponibilidade das tiazidas. As tiazidas podem aumentar os eventos adversos da amantadina. As tiazidas podem reduzir a excreção renal de drogas citotóxicas (por exemplo, ciclofosfamida, metotrexato). Gravidez e lactação: É contra-indicado. Se ocorrer gravidez, telmisartam deve ser descontinuado o mais breve possível. As tiazidas atravessam a barreira placentária e podem causar distúrbios eletrolíticos no feto e é possível que ocorram outras reações. Relataram-se casos de trombocitopenia ou icterícia neonatal com o tratamento tiazídico materno. Reações adversas: Os efeitos adversos observados são, em geral, leves, temporários e raramente requereram a descontinuação da terapêutica. Pode ocorrer diarréia após o uso de telmisartam. Relataram-se casos raros de angioedema, prurido, erupções cutâneas e urticária. Hidroclorotiazida isolada: anorexia, perda de apetite, irritação gástrica, diarréia, constipação, icterícia intra-hepática colestática, pancreatite, sialadenite, xantopsia, leucopenia, neutropenia/agranulocitose, trombocitopenia, anemia aplásica, anemia hemolítica, depressão da medula óssea, reações de fotossensibilidade, febre, erupções cutâneas, reações cutâneas do tipo lupus eritematoso ou reativação urticária, angiite necrotizante, reações anafiláticas, necrólise epidérmica tóxica, sofrimento respiratório, disfunção renal, nefrite intersticial, espasmos musculares, fraqueza, inquietação, visão borrada transitória, escotomas, hipotensão postural, vertigem, parestesia, arritmias cardíacas, distúrbios do sono e depressão. - VENDA SOB PRESCRIÇÃO MÉDICA - MS - 1.0367.0134 - Boehringer Ingelheim do Brasil Química e Farmacêutica Ltda. 